\title{
Wave current interaction: Effect on force prediction for fixed off- shore structures
}

\author{
Mohamed Latheef ${ }^{1, \star}$, Nasir Abdulla ${ }^{2}$, and Mohd Faieez Mohd Jupri ${ }^{2}$ \\ ${ }^{1}$ Department of Civil and Environmental Engineering, Universiti Teknologi Petronas, Tronoh, Perak \\ ${ }^{2}$ PETRONAS Carigali Sdn Bhd (PCSB), Malaysia
}

\begin{abstract}
MetOcean conditions in the South China Sea (SCS) indicates that unlike other locations such as the North Sea, the magnitude of the currents can be relatively large. In addition, these currents are strongly sheared. The present study focused on the typical design problem of calculating the ultimate base shear and overturning moments for slender fixed structures with the inclusion of the interaction between the currents and the wave field. It has been found that the loads on average can be around $15 \%$ larger when this interaction is accounted for in the calculation of the loads, highlighting the importance. In addition, the level of these amplifications were found to be dependent on the sea state steepness and the relative water depth. While no clear trend was found (changed case by case) in the present work, incorporating the vertical structure of the current was found to change the pattern of the amplification of the loads.
\end{abstract}

\section{Introduction}

In the ocean, waves never exist without an associated current. However, as [1] noted, a full description of the flow field that takes into account the interaction between the waves and the current is never undertaken in the design of either coastal or offshore structures. The main reason for this is that in regions of the world where the majority of the offrshore oil explorations have been undertaken, namely, the Gulf of Mexico and North Sea, the magnitude of the current is of the order of tens of $\mathrm{cms}^{-1}$.

In South China Sea (SCS), this is not applicable because the currents are much stronger and remain around the same order of magnitude as the underlying wave kinematics. Therefore, ignoring the effect of wave-current interaction is not a viable option for the design of offshore structures located in SCS. In addition to the large magnitude, the currents in the SCS are strongly sheared. Despite its potential significance, a thorough study of how this may affect the forces on structures located in this region has not been undertaken so far.

It is exactly this problem that this paper will address. More specifically, it will provide the first answer to the question of how significant wave-current interaction is on the predicted magnitude of the forces on fixed offshore structures; the focus on fixed structures stemming from the fact that they are the most common in SCS and the simplest to deal with. To keep in tune with the usual design procedures, the present study will only consider regular waves. This means that the present work only

^Corresponding author:: mohamed.latheef@utp.edu.my 
focuses on the ultimate base shear and overturning moment calculations. Also, the focus will be only on depth uniform currents and linearly sheared currents. This ensures that the analytical potential flow solutions remain valid, providing a simple tool in the calculation of the forces. At this point, it should be stressed that the actual current profile in SCS are strongly sheared and resemble an exponential profile. Despite this, the linear profiles considered in the present work will provide a guidance on how important the vertical structure of the current can be in the typical design calculations.

The paper continues in $\$ 2$, providing the background material relating to the analysis carried out in this paper while $\$ 3$ expands on the methodology adopted in the present study. Section 4 provides the results before the paper concludes in $\$ 5$ with suggestions for future work.

\section{Background}

The present study will be limited to the regular wave loading on fixed slender offshore structures comprising of tubular members. The current section provides a brief background on the methodology to calculate the fluid loading on such structures.

\subsection{Fluid loading}

A tubular member is considered as slender when the diameter to wave length ratio, $D / \lambda \leq 0.2$. For this regime, after [2], the force per unit length, $f_{T}$, is expressed by the sum

$$
f_{T}=\rho C_{M} A \frac{\partial u}{\partial t}+\frac{1}{2} \rho C_{D} D u|u|,
$$

where $C_{M}$ and $C_{D}$ are inertia drag coefficients, respectively, $u$ is the normal component of the water particle kinematics, $\frac{\partial u}{\partial t}$ is the normal component of the fluid acceleration, $D$ is the diamter of the member, $A$ is the area of the member and $\rho$ is the density of the fluid, taken as $1000 \mathrm{kgm}^{-3}$ throughout the present study. The first term in Equation 1 is the inertia component and the second term is known as the drag component.

The relative importance of these two forces are expressed by the nondimensional Keulegan-Carpenter number,

$$
K C=\frac{U T}{D},
$$

where $U$ is a representative fluid velocity and $T$ is the wave period. The drag and inertia cofficients, $C_{D}$ and $C_{M}$ are usually expressed as functions of $K C$. These parametrisations are usually obtained through model testing. For the present study, the values proposed by [9] of $C_{D}=0.65$ and $C_{M}=1.6$ will be used throughout.

\subsection{Water particle kinematics}

Equation 1 is a relatively simple equation to apply. The complexity of the wave loading process is encapsulated in the calculation of the $u$ and $\frac{\partial u}{\partial t}$ terms. There is no limit to the nonlinearity of the wave field that can be included in these terms nor are there any changes requried when the waves are regular or irregular. 


\subsubsection{Fenton (1985) [3] Stokes' $5^{\text {th }}$ order solution}

Stokes' $5^{\text {th }}$ order solution is the most commonly adopted model to calculate the wave kinematics required in the Morrison's equation for the determination of the ultimate base shear and overturning moment of fixed structures. The reason for this is twofold: firstly, being an analytical solution, it is straightforward to implement it and secondly due to the use of so-called loading recipes. Loading recipes is a reference to the phenomenon of obtaining the $C_{D}$ and $C_{M}$ coefficients from model test data by assuming underlying Stokes' fifth order kinematics.

The Stokes' $5^{\text {th }}$ order solution is derived by assuming the flow is irrotational, and thus the existence of a velocity potential, which can be used to describe the 2D flow velocities, $(u, w)$ as:

$$
u=\frac{\partial \phi}{\partial x}, \quad w=\frac{\partial \phi}{\partial z}
$$

The technique used in this family of solutions is the perturbation approach where all the quantities are expressed as a power series of the small parameter, wave steepness, $k H / 2$. This technique allows the derivation of a heirrechy of solutions for each order of wave steepness. While Airy (1845) [4] obtained the linear solution (first order) solution, Stokes (1849) [5] derived a solution correct up to a third order of wave steepness. Since then, many authors have extended the solution to higher orders. Of these, the most widely used form was the one that was derived by [3]. The surface elevation, $\eta$, and the velocity potential, $\phi$ for this solution are expressed as

$$
\eta(x, t)=\sum_{i=1}^{5} \sum_{j=1}^{i} \epsilon^{i} B_{i j} \cos (j(k x-\omega t)),
$$

and

$$
\phi(x, z)=C_{0}\left(\frac{g}{k^{3}}\right)^{\frac{1}{2}} \sum_{i=1}^{5} \sum_{j=1}^{i} \epsilon^{i} A_{i j} \cosh (j k(z+d)) \sin (j(k x-\omega t)) .
$$

In these equations, $k$ is the wave number, $\omega$ is the circular wave frequency, $\epsilon=k H / 2,(x, z)$ refers to the spatial coordinates with $z$ pointing upwards at the mean water level, the coefficients $A_{i j}, B_{i j}$ and $C_{i}$ are functions of $k$ and water depth $d$ and provided in [3].

[3] derived his solution to include a mean horizontal current of magnitude, $U$. With the inclusion of this current, the horizontal velocity, $u$, is given by

$$
u=U+\frac{\partial \phi}{\partial x} .
$$

Before this solution can be applied to the calculation of forces, as a first step, for a given $T, H$ and $d$, $k$ needs to be calculated. This is done by numerically solving

$$
\left(\frac{k}{g}\right)^{\frac{1}{2}} U-\frac{2 \pi}{T(g k)^{\frac{1}{2}}}+C_{0}(k d)+\left(\frac{k H}{2}\right)^{2} C_{2}(k d)+\left(\frac{k H}{2}\right)^{4} C_{4}(k d)=0 .
$$

\subsubsection{Fenton (1988) [6] stream function}

In this solution, the stream function, $\psi(x, z)$, defined such that

$$
u=\frac{\partial \psi}{\partial z}, \quad w=-\frac{\partial \psi}{\partial x}
$$


is expressed as

$$
\psi(x, z)=-\bar{U}(z+d)+\left(\frac{g}{k^{3}}\right)^{\frac{1}{2}} \sum_{j=1}^{N} B_{j} \frac{\sinh j k(d+z)}{\sinh j k d} \cos (j k x),
$$

where $B_{j}$ are unknown coefficients. Similarly, the surface elevation is expressed as a Fourier series with $N$ unknown coefficients. The unknown coefficients in $\psi$ and $\eta$ are obtained through a Newton-Raphson iterative procedure to enforce the fully nonlinear boundary conditions. Again, this solution caters for a depth uniform current of magnitude $\bar{U}$.

\subsubsection{Kishida \& Sobey (1988) [7]}

Lamb (1945) [8] showed that when waves propagate on a linearly sheared current (constant vorticity), the resulting wave field remains irrotational. The resulting flow velocity field can be expressed as

$$
\begin{gathered}
u(x, z)=\Omega_{0} z+U_{s}+\frac{\partial \phi}{\partial x}, \\
w(x, z)=\frac{\partial \phi}{\partial z},
\end{gathered}
$$

where $\Omega_{0}$ is the vorticity, $U_{s}$ is the current velocity at the surface. Using the same perturbation method as [3], [7] obtained an analytical solution for this problem, correct up to the third order of wave steepness. The solution for $\eta(x, z)$ and $\phi(x, z)$ are again provided in a form similar to Equations 4 and 5 . The form of the dispersion equation for this solution is given by

$$
\frac{\omega}{k}=C_{0}\left(a_{11}-\epsilon^{2} a_{20}\right)+U_{M}+\frac{1}{2} \Omega_{0} d
$$

where the coefficients $C_{0}$ and $a_{i j}$ are functions of $k, d$ and $\omega_{0}$ and

$$
U_{M}=\frac{1}{2}\left(U_{s}+U_{b}\right)
$$

where $U_{b}$ is the current velocity at the bed.

\section{Methodology and cases}

To evaluate and quantify the effect of wave-current interaction, the simplest loading scenario for fixed structures was considered. This scenario is the calculation of the total base shear and overturning moment of a single column of diameter, $D$. Given that the basic tubular member is what is used to build up complex fixed offshore structures such as jacket platforms, the conclusions regarding the loading of this single column is directly applicable to the more complex structures. Another simplification adopted in the present study was the exclusion of dynamic response of the column. Again, this is justified based on the fact that the dynamic wave loading forms the input to any complex dynamic analysis. A definition sketch for the problem considered in the present study with the coordinate axes and the placement of column are provided in Figure 1.

To follow the industry practise in the calculation of the total base shear and the overturning moment, the metocean conditions for the 100 year storm events were used. These conditions were chosen for three locations in the SCS and were obtained from [9]. The choice of these locations were only motivated by the need to cover a range of $k d$ and the steepest sea states. The details of the metocean 


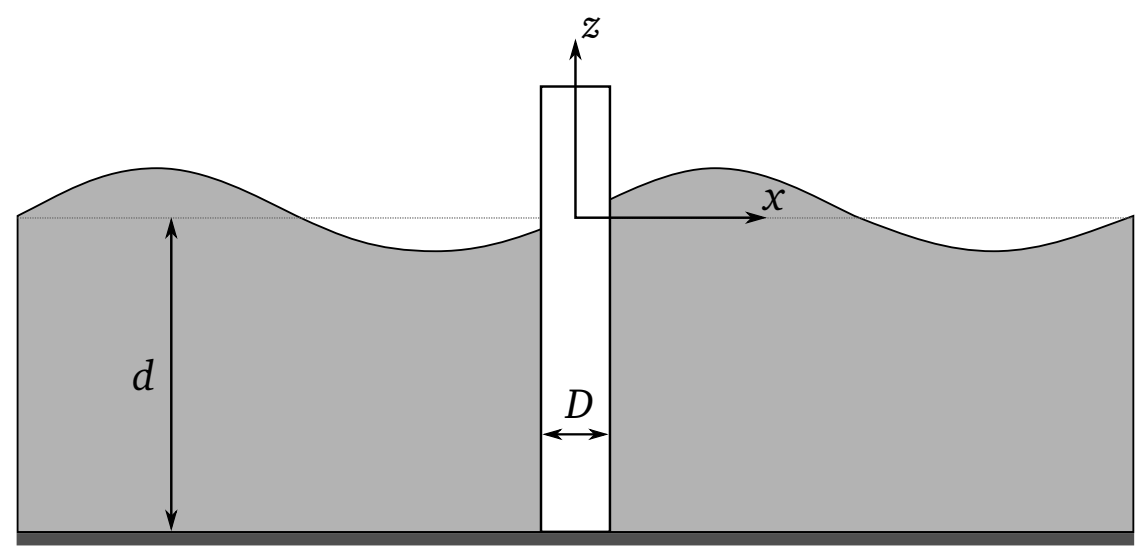

Figure 1. Definition sketch for the calculations, showing the placement of the column

Table 1. Metocean criteria for the locations chosen for analysis

\begin{tabular}{llccc}
\hline Parameter & Unit & Location, L1 & Location, L2 & Location, L3 \\
\hline$H$ & {$[\mathrm{~m}]$} & 11.0 & 10.8 & 14.5 \\
$T$ & {$[\mathrm{~s}]$} & 9.0 & 11.0 & 12.8 \\
$d$ & {$[\mathrm{~m}]$} & 62 & 50 & 50 \\
$U(z=0)$ & {$\left[\mathrm{m} \mathrm{s}^{-1}\right]$} & 2.3 & 1.3 & 1.3 \\
$U(z=-d / 2)$ & {$\left[\mathrm{m} \mathrm{s}^{-1}\right]$} & 1.8 & 1.1 & 1.2 \\
$U(z=-0.01 d)$ & {$\left[\mathrm{m} \mathrm{s}^{-1}\right]$} & 0.5 & 0.7 & 0.7 \\
$k d$ & {$[-]$} & 3.09 & 1.76 & 1.39 \\
$H k / 2$ & {$[-]$} & 0.27 & 0.19 & 0.20 \\
\hline
\end{tabular}

conditions at the chosen locations are summarised in Table 1. For the chosen locations, the relative water depth range from shallow end of intermediate $(k d=1.39$ for L3) to effectively deep $(k d=3.09$ for $L 1$ ) while the wave steepness range from $a k=0.27$ to 0.19 . Hereafter, the wave conditions will be referred to by L1, L2 and L3.

Having chosen the structure and wave cases, the rest of the methodology is simple. A range of the column diameter, $D$, were chosen such that $15 \leq K C \leq 105$ and the total base shear, $F_{B S}$ and overturning moment $M_{O}$ were calculated for each $D$ by integrating Equation 1 as

$$
F_{B S}(t)=\int_{-d}^{\eta(t)} f_{T}(t, z) \mathrm{d} z
$$

and

$$
M_{P}(t)=\int_{-d}^{\eta(t)} z f_{T}(t, z) \mathrm{d} z .
$$

In these calculations, the wave kinematics required to evaluate $f_{T}(t, z)$ were calculated using three methods:

1. Stokes' $5^{\text {th }}$ order solution assuming no wave-current interaction, however, $u=\frac{\partial \phi}{\partial x}+U$ and hereafter denoted as Method 1, M1, 
2. Stream function solution by assuming a depth uniform current, $U_{M}$ and full wave-current interaction, denoted as Method 2, M2, and

3. Kishida \& Sobey (1988) [7], incorporating a linearly sheared current and denoted as Method 3, M3

It should be pointed that Method 1 is a widely adopted simplification used in design. In applying Method 2 and Method 3, how to define $U_{M}$ and $\Omega_{0}$ is a question that needs to be resolved. In the present study, $U(z=-d / 2)$ in Table 1 was used as $U_{M}$ for each location, while $\Omega_{0}$ is defined as

$$
\Omega_{0}=\frac{U(z=0)-U(z=-0.01 d)}{d},
$$

for each location. It should be highlighted that the real current profiles in Table 1 are exponential in nature. The choice for Method 2 is motivated by the fact that using $U_{s}$ instead of $U_{m}$ would lead to an overly conservative estimate of the forces while choice for Method 3 is motivated by the need for the largest value of $\Omega_{0}$.

\section{Results}

For the various combination of $k d$ and $a k$ values, it is important to establish the ideal value of the truncation order, $N$, for the stream function solution provided in Equation 9. To perform this, at successive $N$, the horizontal velocity at the surface over a whole wave period was calculated. When the root mean square (r.m.s) error between successive order reduces to $10^{-6} \mathrm{~ms}^{-1}$, it was decided that the solution has converged. This calculated was performed for a zero current, a co-flowing current and an opposing current. Figure 2 provides the results for these calculations. In all the three subplots, the three MetOcean conditions that have been considered in the present study have been marked. For all three locations, irrespective of the current and its direction, the commonly adopted $5^{\text {th }}$ order solution was found to not have converged. Also, a higher $N$ was required for convergence with the inclusion of the current. Considering the currents further, it was found that a higher $N$ was required for the opposing current. For example, taking L1, which is the steeepest, for no current, it converged at $N=7$, for a co-flowing current at $N=8$ and for an opposing current, at $N=11$. Following these results, to avoid any convergence issues, all the calcultions for $M 2$ were undertaken using $N=15$.

Figure 3 provides the comparison of the variation of the normalized base shear with $K C$ for all three locations with a co-flowing and an opposing current, plotted as solid lines and dashed lines, respectively. For the three subplots, which corresponds to the chosen three locations, the maximum base shear for a given $K C$ that has been calculated by employing $M 2$ and $M 3$ have been normalised by the corresponding maximum base shear for that $K C$, calculated using $M 1$; black lines corresponding to $M 2$ while gray lines correspond to $M 3$. Overall, the calculation of the total base shear using $M 2$ and $M 3$ leads to larger loads compared to the simplified procedure of using the Stokes' $5^{\text {th }}$ order solution (M1) with no consideration of wave-current interaction, ranging from around 5\% to as high as 500\%. Also, this magnification of the loads tie in neatly with the steepness of the sea state under consideration. The steepest sea state corresponding to $L 1$ shows overall, larger amplification compared to $L 2$ and $L 3$. Although the wave steepness at $L 2$ and $L 3$ are comparable, $L 3$ shows larger amplifications (on average 5\% higher). This shows that for a similar steepness, lower $k d$ (shallower) leads to larger amplifications when wave-current interaction is taken into account. Apart from $L 2$, an opposing current leads to larger amplification and more variability in the total base shear with $K C$, compared to a co-flowing current. This is expected because an opposing current will lead to steepening of the waves, which in turn will lead to larger elevations, kinematics and thus loads. 


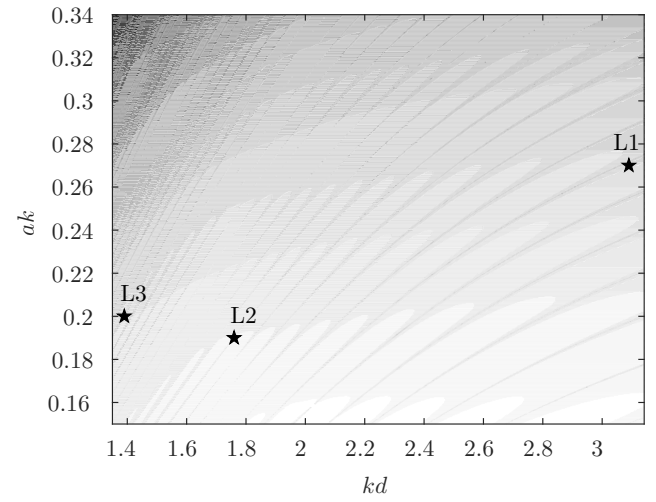

(a)

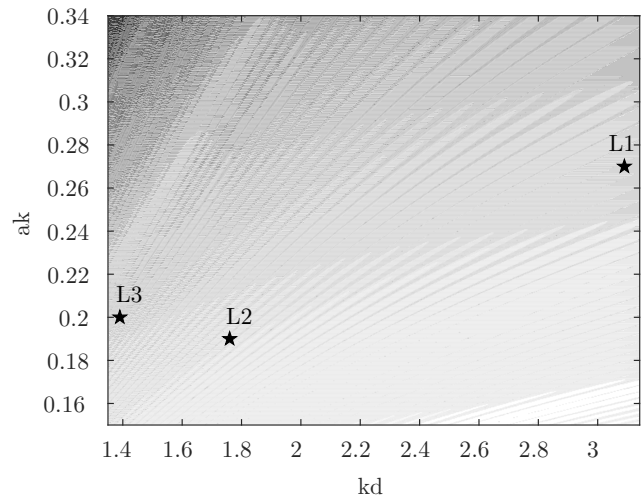

(b)

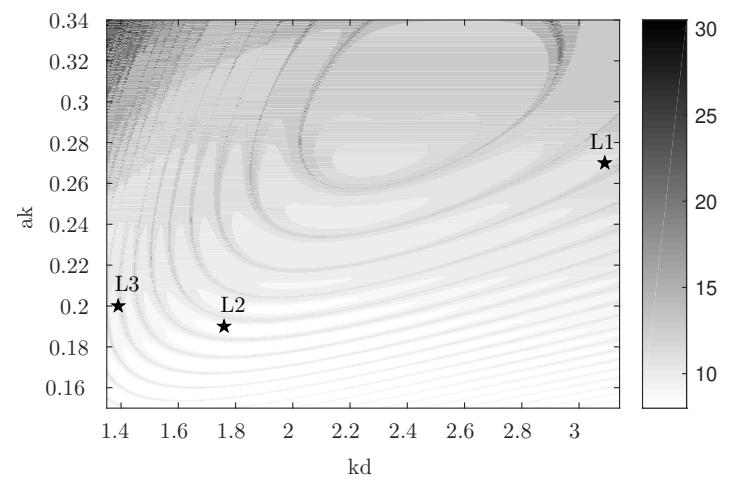

(c)

Figure 2. The variation of the converged truncation order, $N$, with $a k$ and $k d$ for (a) $U=0$, (b) $U=+1 \mathrm{~m} \mathrm{~s}^{-1}$ and (c) $U=-1 \mathrm{~m} \mathrm{~s}^{-1}$.

Considering the differences between $M 2$ and $M 3$, no concrete trend is available, that is applicable to all three locations. However, for all three locations, in the case of a co-flowing current, the force amplifications is higher for $M 2$ compared to $M 3$. This trend is reversed for the opposing current for $L 1$ and $L 3$ with $M 3$ providing larger amplifications compared to $M 2$. It should be pointed that while for $M 2$, a relatively high truncation order $N=15$ was adopted, for $M 3$, the analytical solution is provided only upto a third order. For these comparisons to be like for like, the $M 3$ solution also needs to be calculated at a similar order of wave steepness. However, the present results suggests that the inclusion of the shear in the current profiles remains very important in SCS.

The results of applying the same analysis in the calculation of the base overturning moment is provided in Figure 4. The trends discussed for base shear are applicable to these results, with similar amplifications for each location. The important difference is that, $M 2$ leads to higher amplification compared to M3 in the case of opposing currents, which is the reverse of the trend observed for the base shear. 


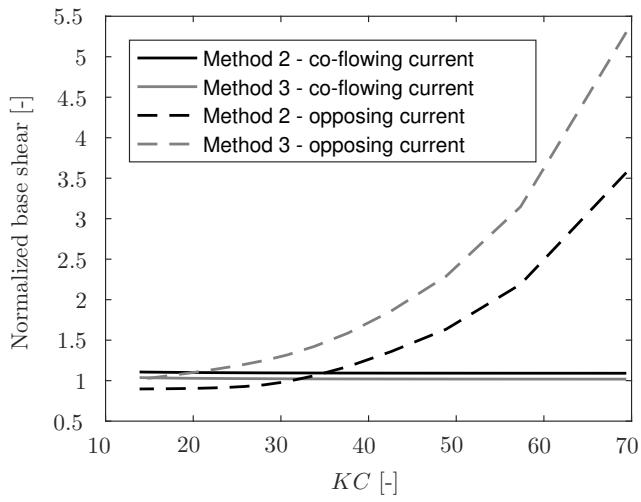

(a)

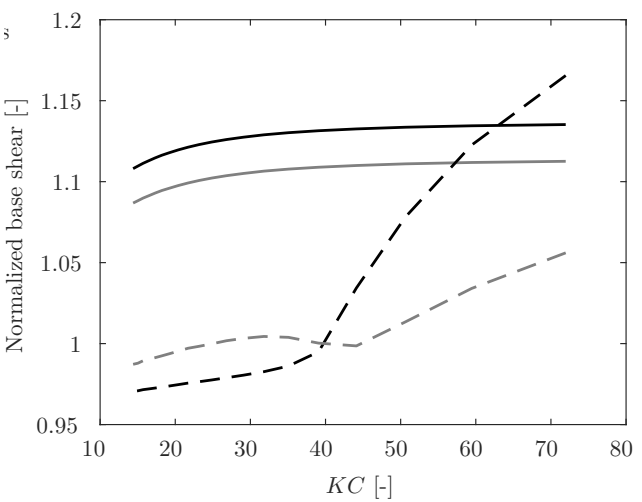

(b)

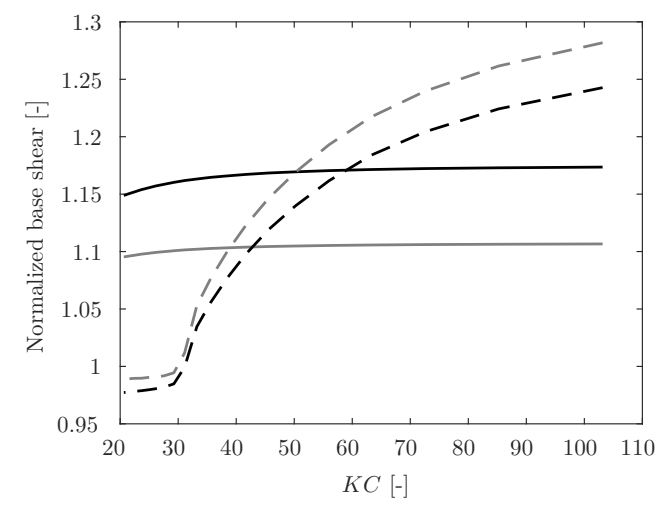

(c)

Figure 3. Variation of the total base shear normalized by the predictions from forces calculated using $M 1$ with $K C$ for (a) L1, (b) L2 and (c) L3.

\section{Conclusion}

The ultimate base shear and overturning moment on a tubular column for varying diameter have been calculated based on the 100 year storm conditions for three locations in the SCS. Constraining the diameter such that the forces are in the slender body loading regime, Morrison's equation have been used in the computation of the forces. Given the strong current present in all three locations chosen for the present study, the wave kinematics used in these computations have been calculated using three methods, two of which considers the wave-current interaction. Of these two methods, one treats the current as depth uniform (denoted as M2) while the other treat it as a linearly sheared current (denoted as M3), which is the more representative case. For both $M 2$ and $M 3$, both a co-flowing and an opposing current were considered.

Comparing the base shear and overturning moment obtained by $M 2$ and $M 3$ to that obtained with the assumption of no wave-current interaction, in general, $M 2$ and $M 3$ leads to larger loads. These amplifications are on average around $15 \%$. It was also found that in general, an opposing current leads to larger amplifications compared to a co-flowing current. Based on the limited number of cases that have been considered in the present study, it is observed that the amplifications are related to the incident wave steepness, $a k$, and the relative water depth, $k d$, with steeper waves and shallower 


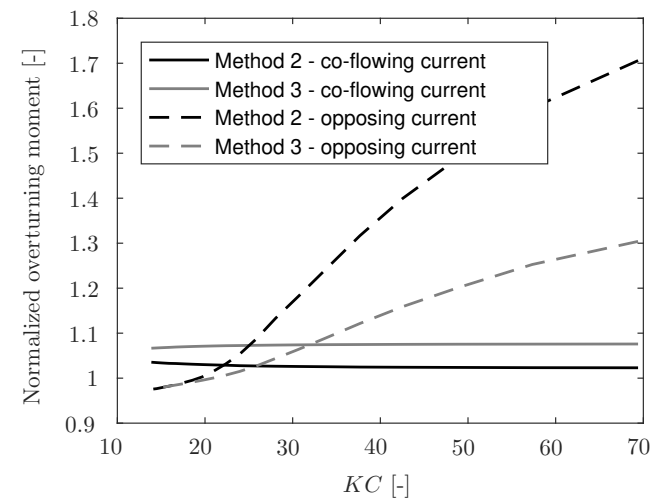

(a)

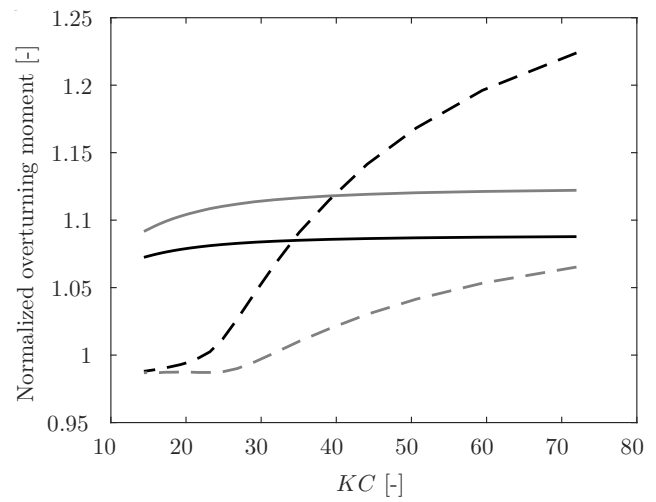

(b)

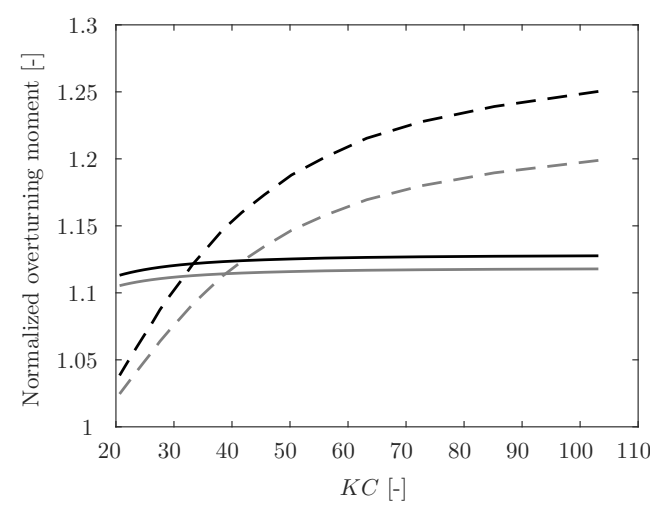

(c)

Figure 4. Variation of the overturning moment normalized by the predictions from overturning moments calculated using $M 1$ with $K C$ for (a) L1, (b) L2 and (c) L3.

water leading to larger amplifications. These results highlight the importance of incorporating the wave current interaction in the safe design of fixed offshore structures.

Considering the form of the current included in the analysis, it was found that $M 2$ and $M 3$ leads to differing loads. In general, it was found that for ultimate base shear, an opposing sheared current leads to larger amplifications while this trend is reversed for overturning moments. Similarly, when the current is co-flowing, M2 leads to larger amplifications while the trend is reversed again for overturning moments. It should be emphasized that while this comparison highlights the importance of the inclusion of the vertical structure of the current, the comparison is not like for like. This is because, $M 3$ relates to a third order solution while $M 2$ has been calculated at a truncation order of 15 .

In the present work, all the calculations have been undertaken for regular waves, following the industry practise for the ultimate base shear and overturning moments. However, real waves are never regular. Hence, these calculations needs to be undertaken for irregular waves propagating on currents. 


\section{References}

[1] G. Thomas, G. Klopman, Wave-current interactions in the near shore region, INTERNATIONAL SERIES ON ADVANCES IN FLUID MECHANICS 10, 255 (1997)

[2] J. Morison, J. Johnson, S. Schaaf et al., The force exerted by surface waves on piles, Journal of Petroleum Technology 2, 149 (1950)

[3] J.D. Fenton, A Fifth-Order Stokes Theory for Steady Waves, Journal of Waterway, Port, Coastal, and Ocean Engineering 111, 216 (1985)

[4] G.B. Airy, On the Laws of the Tides on the Coasts of Ireland, as Inferred from an Extensive Series of Observations Made in Connection with the Ordnance Survey of Ireland, Philosophical Transactions of the Royal Society of London 135, pp. 1 (1845)

[5] G.G. Stokes, On the theory of oscillatory waves, Transactions of Cambridge Philosophical Society 8, 441 (1849)

[6] J. Fenton, The numerical solution of steady water wave problems, Computers \& Geosciences 14, 357 (1988)

[7] N. Kishida, R.J. Sobey, Stokes Theory for Waves on Linear Shear Current, Journal of Engineering Mechanics 114, 1317 (1988)

[8] H. Lamb, Hydrodynamics Dover, New York 43 (1945)

[9] PETRONAS Technical Standards, Design of fixed offshore structures (working stress condition), PTS 34.19.10.30, (2012) 